\title{
SAFE ESTIMATION OF MINIMUM THICKNESS OF CIRCULAR MASONRY ARCHES CONSIDERING STEREOTOMY AND DIFFERENT ROTATIONAL FAILURE MODES
}

\author{
ORSOLYA GÁSPÁR ${ }^{1 *}$, ISTVÁN SAJTOS ${ }^{2}$ AND ANDRÁS Á. SIPOS ${ }^{3}$ \\ 1,2,3 BME Budapest University of Technology and Economics \\ Department of Mechanics, Materials and Structures \\ 1111 Budapest, Muegytem rkp 3., Hungary \\ e-mail:1* gaspar@szt.bme.hu, ${ }^{2}$ sajtos@szt.bme.hu, ${ }^{3}$ siposa@eik.bme.hu, www.szt.bme.hu \\ (*corresponding author)
}

Keywords: masonry arch, catenary, thrust line, pointed arch, minimum thickness analysis, geometric indeterminacy

\begin{abstract}
Limit state analysis of masonry arches sets to assess the safety of the structure by determining the minimum thickness that just contains a thrust line. Based on the Heymanian assumptions regarding material qualities and the equilibrium approach to the static theorem it has been explicitly proven for semi-circular arches that both the thrust line and the resulting minimum thickness value is subject to stereotomy (brick or stone laying pattern), while present study demonstrates, that the latter statement holds for pointed-circular arches as well. This is not straightforward, since the number- and arrangement of the hinges at limit state vary subject to the geometry in case of pointedcircular arches, resulting a more complex problem. It is also explicitly shown, that stereotomy might also affect the corresponding (rotational) failure mode (for certain arch geometries). Stereotomy of an existing structure is not always known, hence it is relevant to search for a stereotomy related bounding value of minimum thickness for each of the various failure modes. The potential of the envelope of resultants as a thrust line (resulting from vertical stereotomy) leading to bounding value minimum thicknesses is discussed: as shown elsewhere it bounds the family of thrust lines, hence leads to an upper bound value of minimum thickness in case of semi-circular arches. It is demonstrated however, that this cannot be generalized for other rotational failure modes which occur for circular-pointed arches. The envelope of resultants does not necessarily lead to a bounding value of minimum thickness, and even if it does, it can be either an upper or a lower bound. However, it is found that the range of minimum thickness values is bounded in all possible failure mode types. The necessary conditions are provided for each.
\end{abstract}

\section{INTRODUCTION}

Minimum thickness analysis is based on the consideration, that equilibrium of a structure made of no-tension material is only achievable if the thrust line does not exit its boundaries. It determines the smallest possible cross section, that can still fully incorporate the corresponding 
thrust line for a given loading and compares it to the actual thickness of the arch. Thrust line is subject to the overall geometry of the structure, its loading and its stereotomy (brick or stone laying pattern). The classical approach (e.g. [1],[2]) for minimum thickness analysis assumes a certain stereotomy (mostly radial) and determines the unique thrust line and/or minimum thickness value for a fixed loading. Moseley, however, already suggested [3] that this set up can be varied. Present study considers the relation of thickness and thrust line fixed (that the latter does not exit the boundaries) and seeks a suitable stereotomy for which the thickness becomes minimal (at which the structure turns into a mechanism). This approach was already applied by the authors in investigating the effect of stereotomy on the minimum thickness of semi-circular arches [4]. It has been shown there and elsewhere [5, 6], that for self-load, the envelope of the resultant force vectors coincides with the thrust line in case of a vertical stereotomy: this is called the catenary-type thrust line. It is rather easily comprehensible that it bounds all other thrust lines corresponding to the same thickness and different stereotomy, if the arch's reference line is convex, see also [4]. Consequently, the catenary type thrust line results in an upper bound minimum thickness value for the semi-circular arch.

Even if the Heymanian assumptions are obeyed, the kinematically admissible (rotational) failure modes (number and arrangement of hinges) vary subject to arch geometry [7, 8, 9]. Nikolic's recent rigorous study [8] on the rotational failure modes of pointed arches provided a reportedly inclusive list of all (altogether 5, see Figure 1) possible hinge arrangements subject to the arch geometry. His study suggests, without explicit proof, that 7 is the maximum number of concurrent hinges at the limit state for pointed arches. An analytical proof about that maximum was providec results by investigating circular arches. Steredto search for a stereotoni failure modes, if they ex stereotomy) leading to bounding value minimum thicknesses is discussed, with special

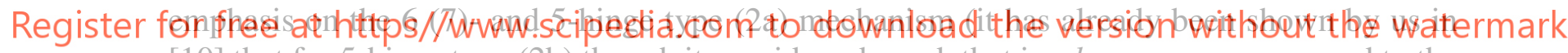
[10] that for 5-hinge type (2b) though it provides a bound, that is a lower one, as opposed to the case of 5-hinge type (1), the semi-circular arch (Figure 1.)). The complexity of the problems discussed here originates in the geometric indeterminacy of the studied mechanisms: since no hinge is located at either the top (6-h) or the bottom(5-h2a) of the arch, the location of the array of resultants might vary relative to the axis of the arch.

Motivation of present study is further discussed in Section, 2. Modelling, geometry and methodology is treated in Section 3, while results on the effect of stereotomy on the minimum thickness value for pointed arches corresponding to the 6-hinge and 5-hinge type (2a) mechanism are derived in Section 4. Finally, conclusions are drawn in Section 5. 

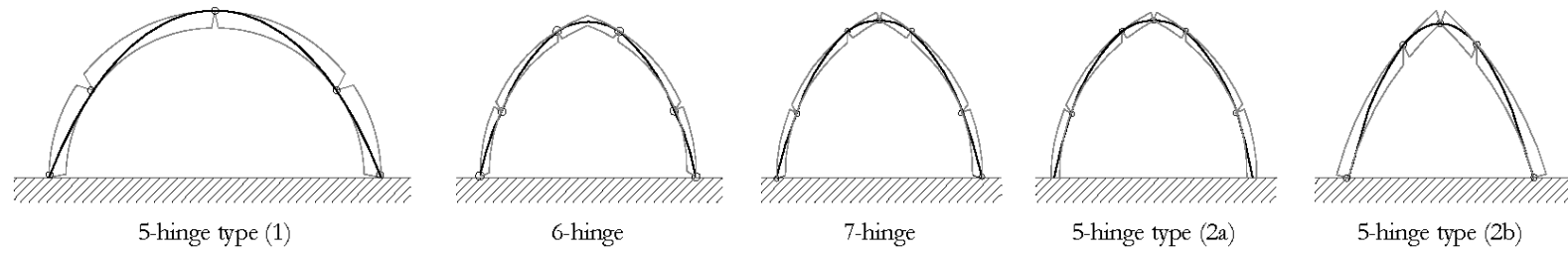

Figure 1. Rotational collapse modes of circular arches, based on [8]

\section{MOTIVATION}

In particular, we aim to investigate, whether

- the dependence of minimum thickness value on stereotomy is valid for all type of rotational failure modes.

- stereotomy affects the type of failure mode.

- it is relevant to define the stereotomy related bounding value of the minimum thickness for each of the various failure mode types (i.e. arch geometry), if exists. It would substantially simplify the analysis if there was one specific type of the thrust line (i.e. stereotomy), resulting a bounding value for all cases. The catenary type

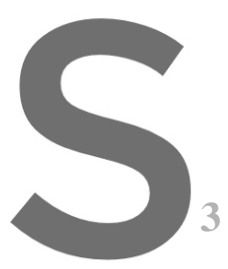
thrust line (
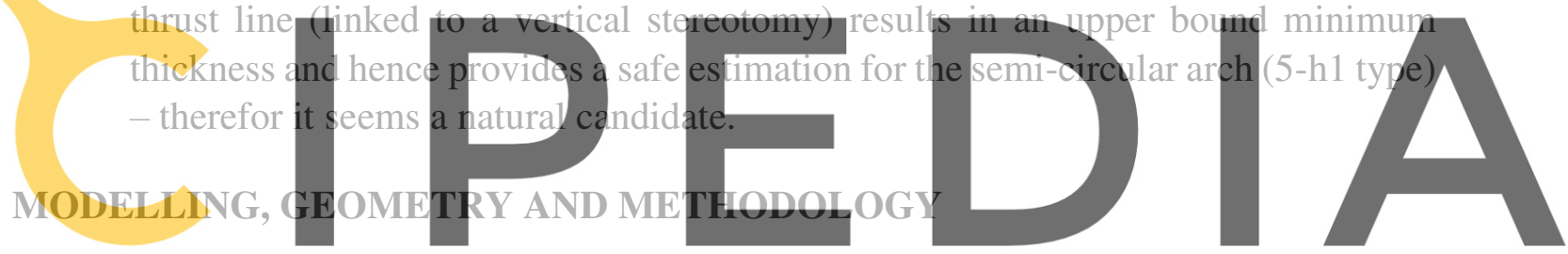

The applied material model is rigid-plastic, furthermore necessary assumptions proposed by

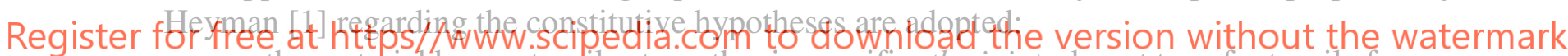
- the material has no tensile strength - in specific, the joints do not transfer tensile forces,

the material has infinite compressive strength,

- no sliding occurs between the elements.

The most notable consequence of these assumptions for present research is that only a rotational failure mode is considered. The static approach of limit state analysis is followed. The equilibrium condition is that the thrust line should not exit the boundaries of the arch. The yield condition is determined by the eccentricity of the resultant force vector: a plastic hinge forms, if the eccentricity equals half-thickness. Following Heyman's proposal [1], minimum thickness analysis is treated as a plastic design methodology that seeks an optimal geometry. The assumed corresponding rotational failure mode (subject to geometry and loading) determines the arrangement of (plastic) hinges at the limit state, which allows for a geometric formulation of the equilibrium and the yield condition: at the limit state the thrust line crosses all hinges (yield) and must be tangent to the boundary of the arch at the internal hinges(equilibrium). In the following, the equilibrium condition is hence denoted tangency condition, in accordance with the geometric formulation. 


\subsection{Geometry}

The arch is modeled as a planar structure made of voussoirs. In order to account for all the theoretically possible arrangements, a continuous model is applied with sections $(s)$ essentially substituting joints. Only constant thickness $(t)$ and symmetrical arches are considered, the analysis is hence carried out on a half-arch and symmetry is accounted for in the constraints. (Minimum) thickness is scaled by the radius $(R)$ of the arch, hence the notation $t / R$ is used. A major simplification of the applied model is that it considers the loading (self-weight) distributed evenly along the center line of the arch, which is treated as the reference line $(r)$, regardless of the stereotomy. Consequently, the $\mathbf{E}$ vector (resultant force, see Figure 3) field becomes independent of the stereotomy and it seems essential for an

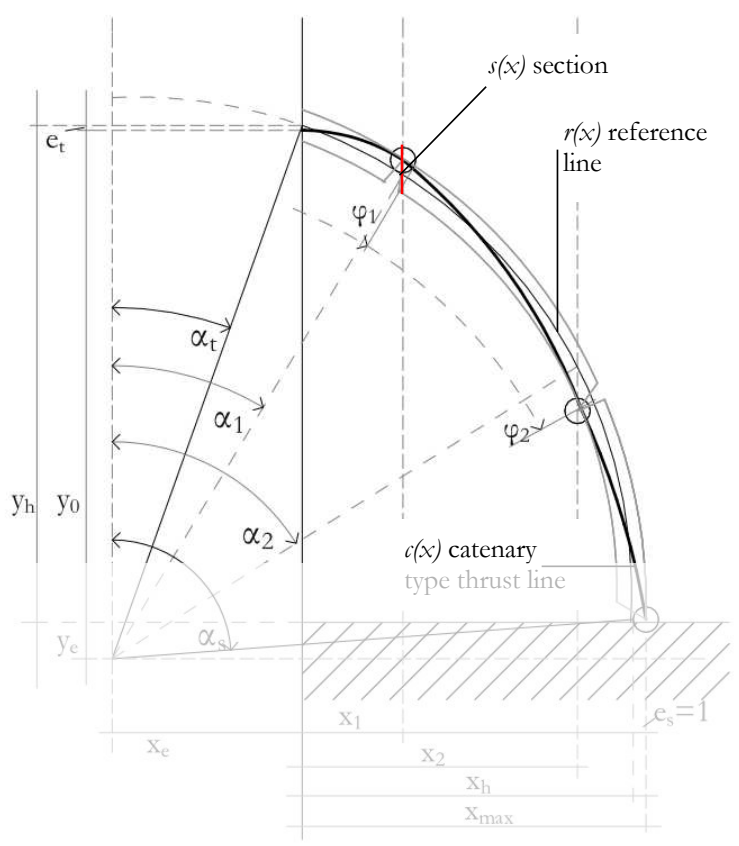
analytical study of the problem.

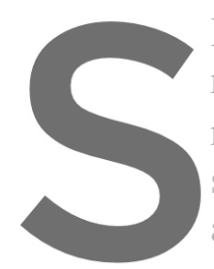
Depending on the problem reference line is either parameterized with respect to the angle to the vertical denoted by $\alpha$ system. Stereotomy is treated as a continuous 1 un along $x$. The thrust line shown in Figure 2 and 3 , right.
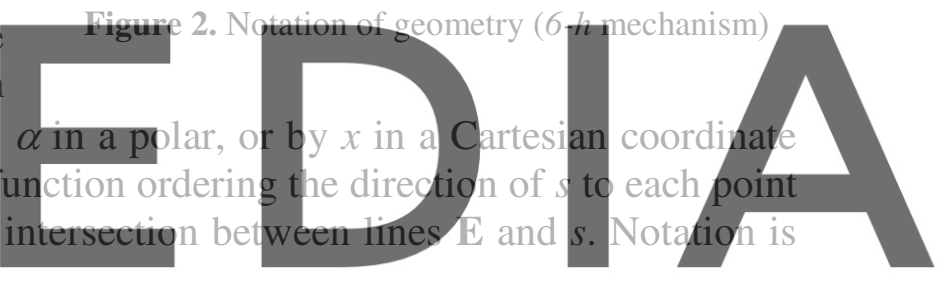
We consider circular-pointed arches with a unit radius $\left(x_{e}, y_{e} \in[0,1]\right)$. We define a deviation

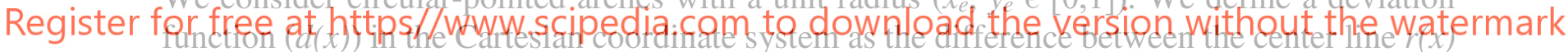
and the catenary-type thrust line $c(x)$. For a catenary-type thrust line results from a vertical stereotomy, $d(x)$ is rather convieniently defined, the applied transformation from the poiar system back and forth is straightforward. Depicting $d(x)$ corresponds to the vertical projection of $c(x)$ and $r(x)$.

$$
d(x):=r(x)-c(x)
$$

The equation of the envelope of resultants (or the catenary-type thrust line) is obtained based on the vanishing moment:

$$
c(x)=y_{0}-\overbrace{\frac{\overbrace{V(x)}(x)}{H}}^{m}\left(x-x_{V}(x)\right),
$$

where $y_{0}$ denotes the $y$-intercept of $H$ at the top, and $x_{V}$ the $x$-coordinate of the center of mass. While $r(x)$ reads

$$
r(x)=\sqrt{1-\left(x+x_{e}\right)^{2}}-y_{e} .
$$

Thrust line's eccentricity at the top from $r(x)$, denoted $e_{t},[-1,1]$ is a unitless quantity, scaled by $t / 2 R$, half-thickness to radius ratio of the arch. Its eccentricity from $r(x)$ at the springing is $e_{s}$ [- 
1,1], analogously to $e_{t}$. At the intersections of $\mathbf{E}(x)$ with the intrados (or extrados) (Figure 2), the following relations hold (the necessary conversion for angles measured at the extrados is made, though not detailed here, before depicting them on the $\alpha-\varphi$ plane for $\varphi$ refers to angles measured at the intrados, see Figure 2, 3):

$$
\begin{aligned}
& x=\left(1 \pm \frac{1}{2} \frac{t}{R}\right) \sin (\varphi)-\sin \left(\alpha_{t}\right), \\
& y=\left(1 \pm \frac{1}{2} \frac{t}{R}\right) \cos (\varphi)-\cos \left(\alpha_{s}\right) .
\end{aligned}
$$

\subsection{Methodology}

The methodology of determining the admissible stereotomy functions for a given minimum thickness value is presented in detail in [4] for the case of the semi-circular arch. A very brief outline is given here, while its application to the more general case of pointed arches is to be found in Section 4. As per definition, the stereotomy-dependent thrust line points lay on the resultants (which only depend on the geometry, i.e $r$ and $t / R$ in the frame of the model), hence those sections of the resultants are inadmissible which are outside (if the arch is sufficiently thin, see [4]) the boundaries of the arch. This allows for the definition of inadmissible ranges of the angle $\varphi$ for each point along the reference line. Here $\varphi$ denotes the internal angle to the vertical of intersection of section and intrados. The results are depicted on the $\alpha-\varphi$ plane for a

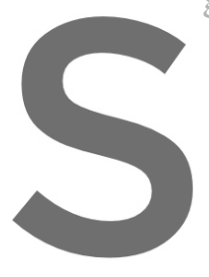
given $t / R$ ratio. A visual explanation is given in Figure 3. below:
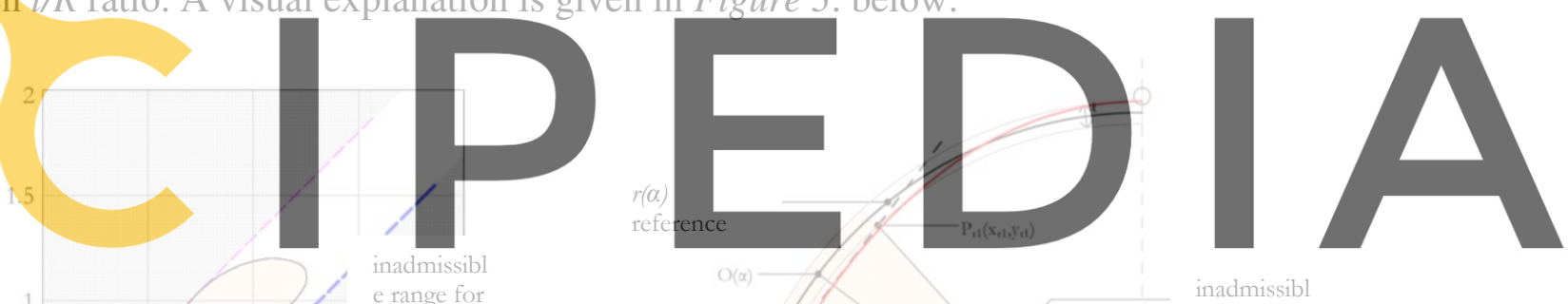

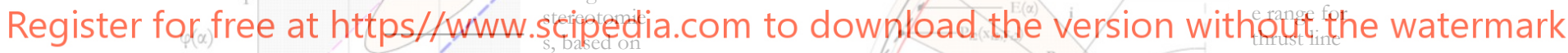
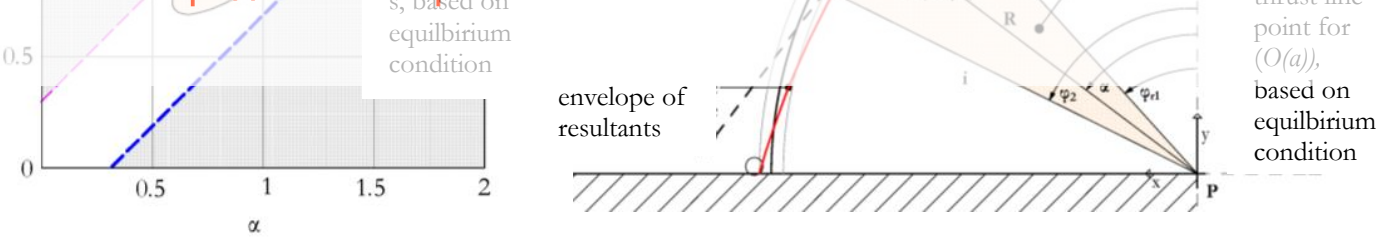

Figure 3. Diagram of admissible stereotomy function-range (left white) in the $\alpha-\varphi$ plane (left), and the explanation of admissible-and inadmissible (orange) ranges on the arch (right)

\section{EFFECT OF STEREOTOMY ON THE MINIMUM THICKNESS VALUE}

Following the initial assumption that the catenary type thrust line leads to a bounding value minimum thickness we start each analysis of an arch (characterized by $x_{e}, y_{e}$, or, equivalently, by $\alpha_{s}$ and $\alpha_{t}$, see Figure 2) with a given $r(x)$ reference line by determining the $t / R$ and the corresponding $\alpha-\varphi$ diagram depicting the admissible range of stereotomies based on $c(x)$. Determining the minimum thickness value of a pointed-circular arch of given geometry $\left(x_{e}, y_{e}\right)$ for a specific stereotomy is done by solving the following system of non-linear equations: 


$$
\begin{gathered}
d\left(x_{1}\right)=c\left(x_{1}\right)-r\left(x_{1}\right)=t_{p r} / 2 R \\
d^{\prime}\left(x_{1}\right)=c^{\prime}\left(x_{1}\right)-r^{\prime}\left(x_{1}\right)=0 \\
d\left(x_{2}\right)=c\left(x_{2}\right)-r\left(x_{2}\right)=-t_{p r} / 2 R \\
d^{\prime}\left(x_{2}\right)=c\left(x_{2}\right)-r\left(x_{2}\right)=0
\end{gathered}
$$

With $x_{1}$ and $x_{2}$ denoting the $x$-coordinate of the internal hinges, and $t_{p r}$ denoting the projection of the half-thickness on the corresponding section. Note that except for the 5-hinge type (1) and (2b) (which are not considered here, see Introduction) always two internal (i.e. between the top and the springing of the arch) hinges appear, one at the intrados, one at the extrados (Figure 1). Then, if possible, we modify the value of $t / R$ along with the $e_{t}$ and $e_{s}$ parameters (see Figure 2, note that $r(x)$ is unaffected!) and observe the effect on the range of admissible stereotomies (essentially the /modified/ $\alpha-\varphi$ diagram) and the rotational failure mode, if applicable. Since the equilibrium related constraints are required, we derive the envelope of resultant for each new combination. It is important to highlight that we only aim to prove that other stereotomies (and hence minimum thicknesses) are possible, but we do not pursue to derive them for illustration. This also holds for the bounding value minimum thicknesses: we only intend to find the combination that results them (in terms of $e_{t}$ and/or $e_{S}$ ) and not the corresponding stereotomy (unless, the bounding value is indeed based on the catenary type thrust line).

Results based on eqs. (6-9) considering the catenary type thrust line for a selected arch geometry (i.e. failure mode 6-hinge) and its corresponding $\varphi$ - $\alpha$ diagrams are depicted in Figure 4, second row, left. Analyzing the the $t / R$ value be higher, ranges corresponding to admissible stereotomy $t / R$ exists, see Figure 4 , first ror their 'geometric indeterminacy' (parameter $e_{t}$ or $e_{s}$ influencing the location of the array of

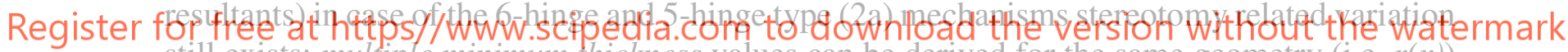
still exists: multiple minimum thickness values can be derived for the same geometry (i.e. $r(x)$ ) and moreover the minimum thickness value derived based on catenary-type thrust line does not provide a bound.

\subsection{6-hinge mechanism}

In the case of a 6-hinge mechanism we have 4, linearly independent unknowns for a given geometry (fixed $x_{e}$ and $y_{e}$ ): $t / R, e_{t}$, and the locations of the internal hinges, $x_{1}$ and $x_{2}$. Consequently, if vertical stereotomy is considered (equivalently: catenary type thrust line), solving eqs. (6-9), results a unique ' $e_{t}$ ' value in addition to the location of hinges and $t / R$ value for a given geometry. However, relaxing the boundary conditions of the minimum thickness analysis still based on catenary-type thrust line (by instead of eqs. (6) and (8) enforcing solely the following inequalities on the problem:

$$
\begin{gathered}
d_{e}\left(x_{1}\right) \leq t_{p r} / 2 R \\
d_{i}\left(x_{2}\right) \leq-t_{p r} / 2 R
\end{gathered}
$$

respectively, opens up a range of admissible values of ' $e_{t}$ ', and allows for stereotomy related variation of minimum thicknesses for the same geometry. Geometrically, condition (11) means 
that we allow the catenary type thrust line to cross the boundary towards the intrados, while condition (12) means it might not even touch extrados (see Figure 4 second row, middle, left and Figure 5). Observe that if both is fulfilled simultaneously alternate admissible stereotomies might exists, as all other members of the thrust line family is 'above' the catenary-type - i.e. one can be found that touches both extrados and intrados. The following questions should then be investigated:

- what is the admissible range of ' $e_{t}$ '?

- whether an analysis based on the catenary-type thrust line results a bounding minimum thickness value?

The deviation function $d(x)$ provides an illustrative tool well suited to investigate these questions:

$$
d(x)=\underbrace{y_{0}-m(x)\left(x-x_{V}(x)\right)}-\overbrace{\sqrt{1-\left(x_{e}-x\right)^{2}}-y_{e}}^{r(x)} .
$$

$r(x)$ depends on the geometry, hence any changes of the value $t / R$ or $e_{t}$ affects $c(x)$ only. $x, x_{v}$ are also independent of these parameters. In order to highlight the effect of any change in these parameters, the following formulation is obtained:

$$
d(x)=\underbrace{\overbrace{\left(y_{h}-y_{e}+e_{t} \frac{t}{2 R}\right)}^{y_{0}})\left(1-\frac{V(x)}{V\left(x_{h}\right)\left(x_{\max }(t / R)-x_{V}\left(x_{h}\right)\right)}\left(x-x_{V}(x)\right)\right.}_{c(x)}-\overbrace{\sqrt{1-\left(x_{e}-x\right)^{2}}-y_{e}}^{r(x)}
$$
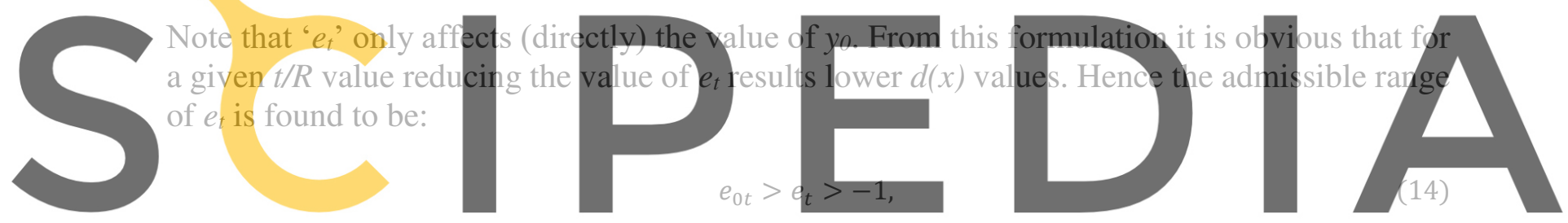

Register for fre et dentes the eccentricity calculated during the determination of minimum thickness intrados, at a fixed $t / R$. Hence, it is theoretically possible, to construct arches (by finding admissible stereotomies) for both higher and lower $t / R$ values than that defined based on the catenary type thrust line: the corresponding lower and upper bound minimum thickness values can be found based on the assumption that $e_{t}=-1$.

$t / R$ can be increased as long as $\min (d(x)) \leq t / 2 R)$, whereas it can be reduced as long as $\max (d(x))$ $\leq t / 2 R)$ - meaning that the envelope of resultant at least touches the intrados, and at most it touches the extrados. The extremities of the range of minimum thickness values hence can be found by solving

or

$$
d_{e}\left(x_{1}\right)=0 ; d^{\prime}{ }_{e}\left(x_{1}\right)=0 ; d_{i}\left(x_{2}\right)=0 \text { while } e_{t}=-1
$$

$$
d_{i}\left(x_{2}\right)=0 ; d^{\prime}{ }_{i}\left(x_{2}\right)=0 ; d_{e}\left(x_{1}\right)=0 \text { while } e_{t}=-1
$$

The former resulting lower, the latter the upper bound. Note that the case of $\boldsymbol{e}=-\boldsymbol{1}$ turns the originally 6-hinge mechanism into a 7-hinge mechanism (see Figure 5, far left and left). 


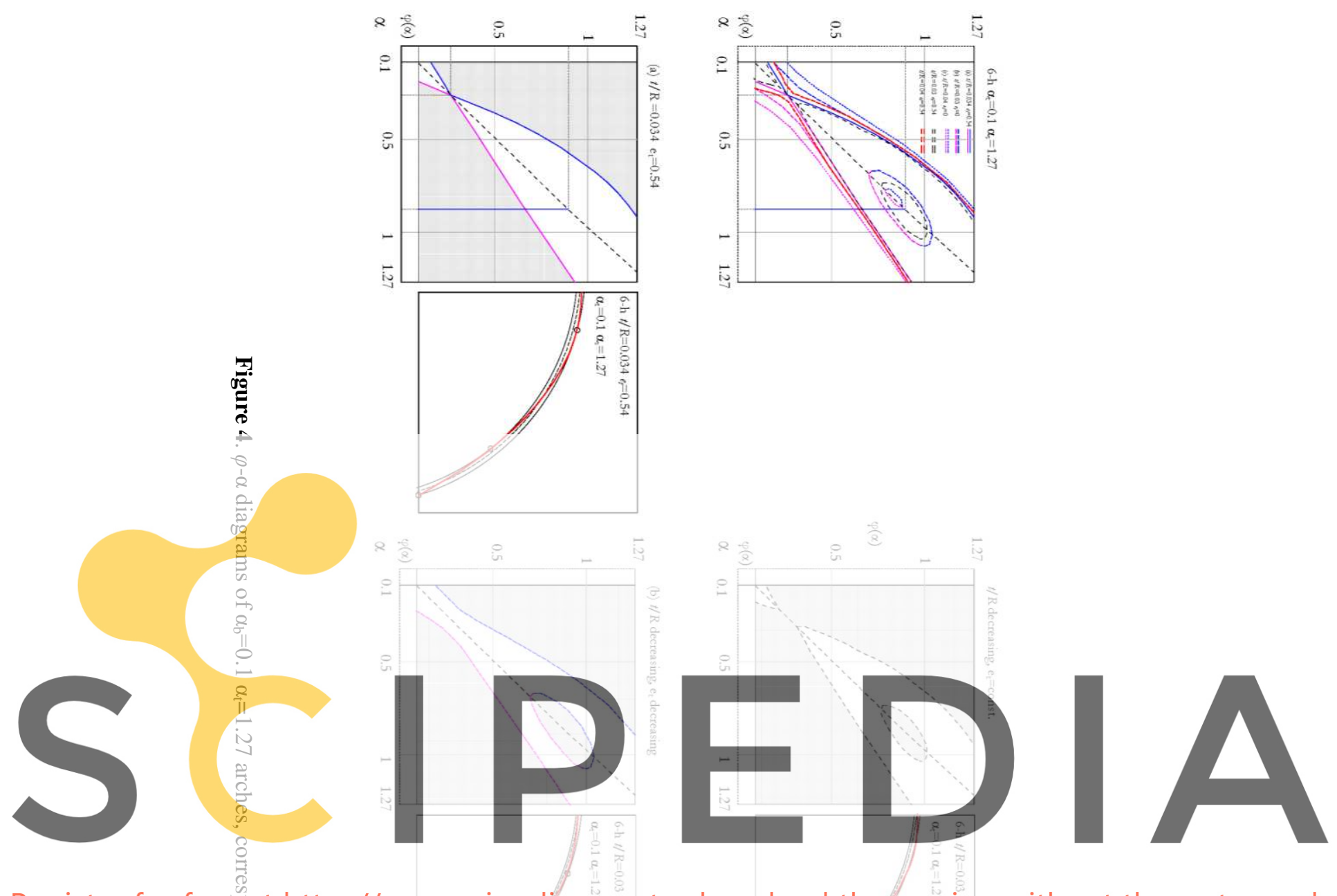

Register for free
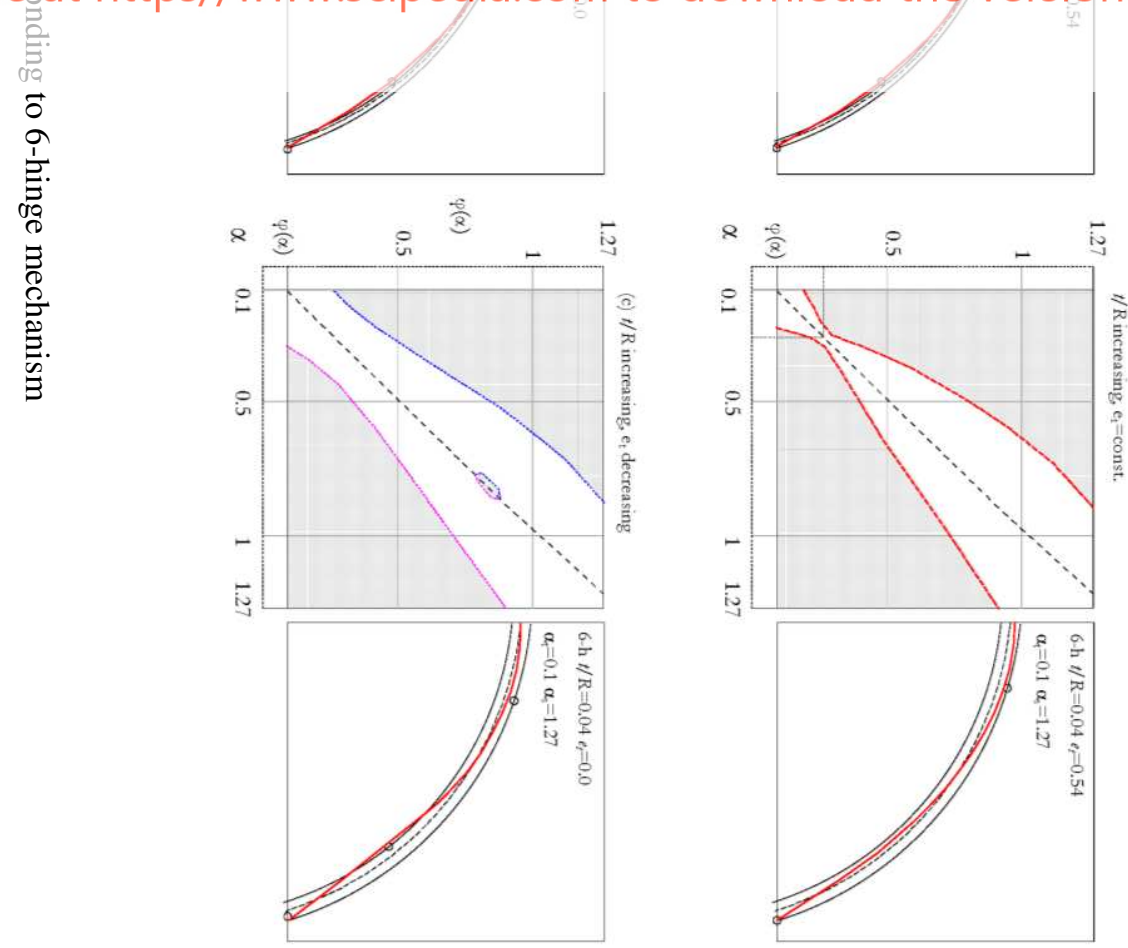


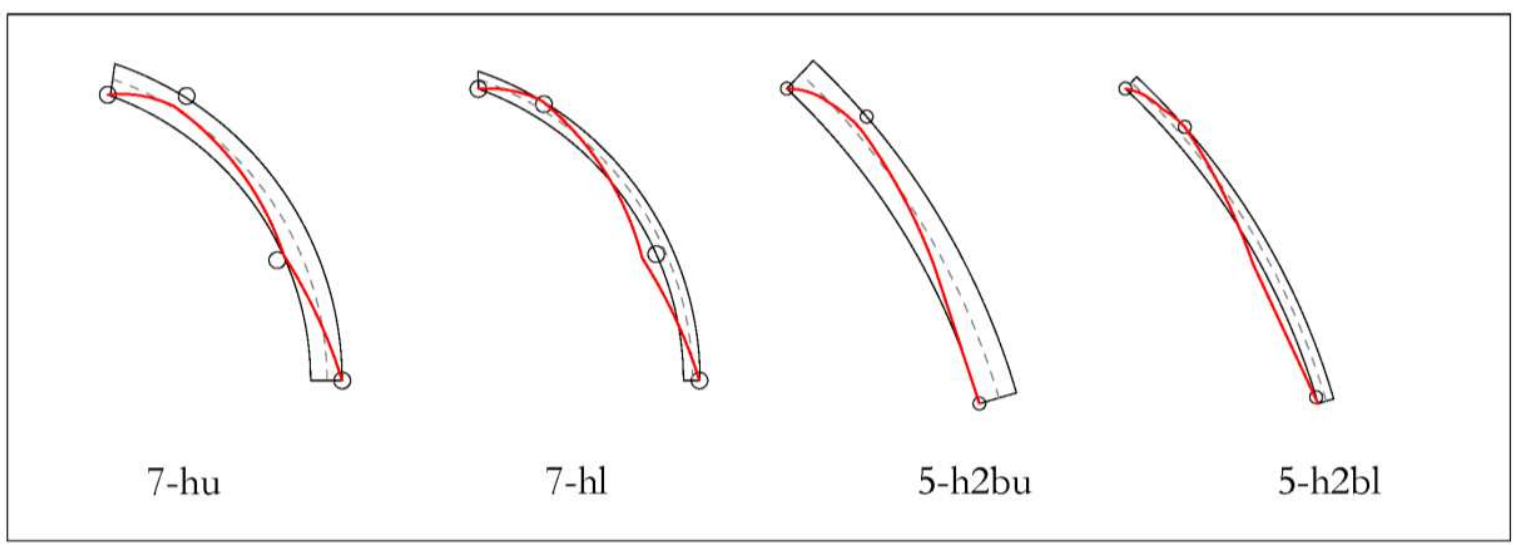

* distorted images for clarity

Figure 5. Hinge arrangement and catenary type thrust line in corresponding limit thickness arches (admissible stereotomy can exist, since all other members of the thrust line family lay above the catenary type thrust line)

Up to this point it has been implicitly assumed that the failure mode is not subject to stereotomy - i.e. only 6-hinge (or in the limit, 7-hinge) mechanisms has been considered. This kept the value of $e_{s}$ fixed, at 1 . However, this is not a necessary condition. Decrease in the value of $e_{s}$ has a similar, reducing effect on $d(x)$ (see below), and it can be activated, if the limit in terms of $e_{t}$ is achieved (i.e. hinge at the top is at the intrados). This turns the 6-hinge mechanism into a 5-hinge type (2a) or

4.2 5-hinge type (2a)
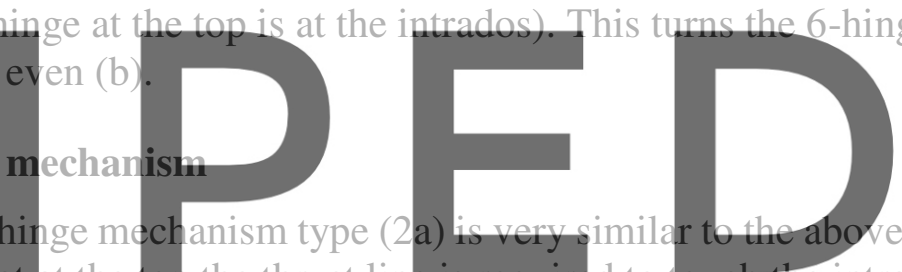

mechanism, except that at the top the thrust li

a hinge at the springing is not a necessary condition, hence $e_{s}$ becomes a parameter. Therefor

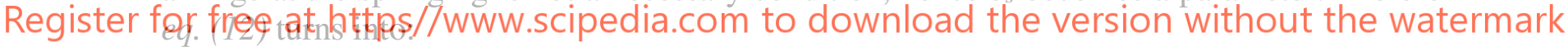

$$
d(x)=\underbrace{\left(y_{0(t / R)}\right)\left(1-\frac{V(x)}{V\left(x_{h}\right)(\underbrace{\boldsymbol{x}_{\boldsymbol{h}}-\boldsymbol{x}_{\boldsymbol{e}}+\boldsymbol{e}_{\boldsymbol{s}} \frac{\boldsymbol{t}}{\mathbf{2 R}}}_{\boldsymbol{x}_{\max }}-x_{V}\left(x_{h}\right))}\left(x-x_{V}(x)\right)\right.}_{c(x)}-\frac{r(x)}{\sqrt{1-\left(x_{e}-x\right)^{2}}-y_{e}}
$$

Clearly, the $e_{s}$ parameter only affects the value of $x_{\max }$. Note that reducing the value of $e_{s}$ results similarly to above lower $d(x)$ values for arbitrary $x$. Following the same argument (see Figure 5 right and far right) as for the case of a 6-hinge mechanism it is deductible that a minimum thickness analysis based on catenary type thrust lines does not provide any bound for the 5 hinge mechanism type (2a) either (either lower or upper). The admissible range of ' $e$ ' is analogously:

$$
e_{0 s}>e>-1
$$

Note that the case of $e_{s}=-1$ turns the originally 5-hinge type (2)a mechanism into a 5hinge type (2b) mechanism (Figure 5). 


\section{CONCLUSIONS}

The most important conclusion of the study above, is that eminently the geometry of the circular pointed arch determines the failure mode, however, it can be modified by the stereotomy, if the failure mode had a geometric indeterminacy (6-hinge, 5-hinge type (2a)) and/or redundancy (7hinge - this, though not explicitly discussed above, is directly deductible from the cases 6-h and 5-h2a). Furthermore, determination of bounding value of minimum thickness for any geometry is possible if the geometric indeterminacy or the redundancy is resolved. To allow comparison with former results of the literature the outcome of the present work is visualized for a given $y_{e}(0.3)$ value in Figure 6: it contains the resulting minimum thickness values with respect to $x_{e}$, for admissible stereotomies. The diagram can be obtained based on eqs. (6-9). It is clearly demonstrated that in perfect agreement with the findings of Nikolić [8] the 7-hinge mechanism marks a local minimum on the $t / R\left(x_{e}\right)$ function considering both the catenary type thrust line, marked bold red or radial stereotomy (marked bold black) in Figure 6. However, if the effect of stereotomy is fully accounted for, the geometry corresponding to the 7-hinge mechanism no longer marks any local minimum. Instead, the observation can be made that lower bound minimum thickness value is to be defined based on the assumption of a 5-hinge type (2b) failure mode. It is important to highlight though that the bounding value minimum thickness (and hence, failure modes) would require unrealistic stereotomies.

The relation of the bold black and red line in the Figure also suggests a very important conclusion for realistic stereotomies. The formerly proven statement on how considering the

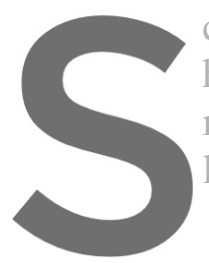
catenary type thrust hinge type (2b) and upp modes with geometric is lower bound for 5-hinge
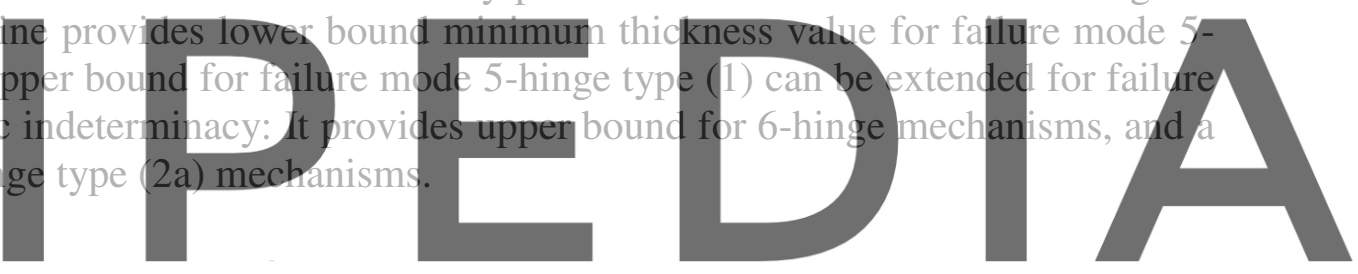

Register for free at htyps//www.scipedia.com to download the version without the watermark

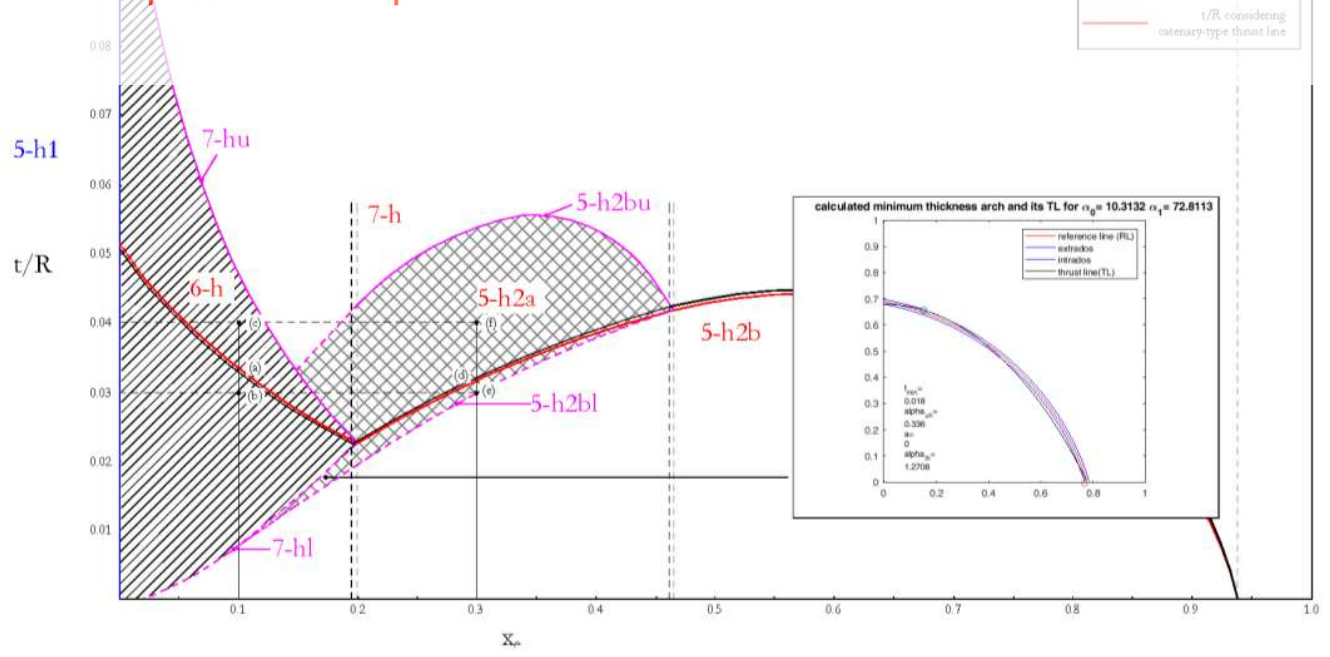

Figure 6. Range of minimum thickness values for $y_{e}=0.3$ and the corresponding rotational failure mechanisms 
Acknowledgements. The research was supported by the NKFIH Grant 124859 and the FIKP grant of EMMI in the frame of BME FIKP-VÍZ.

\section{REFERENCES}

[1] Heyman, J. The safety of masonry arches. International Journal of Mechanical Sciences, 11(4) (1969) pp. 363-385.

[2] Cocchetti, G., Colasante, G., Rizzi, E. On the analysis of minimum thickness in circular masonry arches. Applied Mechanics Reviews,64(5) (2011): 050802.

[3] Moseley, H. On the theory of the arch. In: The theory, practice and architecture of bridges, ed. J. Weale. London: Architectural Library, (1843).

[4] Gáspár, O., Sipos, A. Á. and Sajtos, I. Effect of stereotomy on the lower bound value of minimum thickness of semi-circular masonry arches. International Journal of Architectural Heritage, (2018) pp. 1-23.

[5] Milankovitch, M. Theorie der Druckkurven. Zeitschrift für Mathematik und Physik 55 (1907) pp.1-27.

[6] Alexakis, H.,Makris, N. Limit equilibrium analysis of masonry arches. Archive of Applied Mechanics, 85(9-10) (2015) pp. 1363-1381.

[7] Romano, A., Ochsendorf, J. A. The mechanics of gothic masonry arches. International Journal of Architectural Heritage, 4(1) (2009) pp. 59-82.

[8] Nikolić, D. Thrust line analysis and the minimum thickness of pointed masonry arches. Acta Mechanica, 228(6) (2017) pp. 2219-2236.

[9] Aita, D., Barsotti, R., Bennati, S. Looking at the collapse modes of circular and pointed masonry arches through the lens of Durand-Claye's stability area method. Archive of Applied Mechanics (2019), pp. 1-18.

[10] Gáspár, O., Sipos, A. Á., \& Sajtos, I. The role of rotational collapse mode and catenarytype thrust lines in the limit state analysis of masonry arches. In Proceedings of IASS Annual Symposia 18 (2019), International Association for Shell and Spatial Structures (IASS), pp. 1-8. 